\title{
ENGEVISTA
}

Página da revista: http://www.uff.br/engevista/seer/

\section{Simulação da secagem da soja com coeficiente de difusão variável}

\author{
Mayara dos Santos Zanardi ${ }^{1}$ \\ Leticia Fabri Turetta ${ }^{2}$ \\ Andréa Oliveira da Costa ${ }^{3}$ \\ Esly Ferreira da Costa Jr. ${ }^{4}$
}

Resumo: A soja necessita ser desidratada após a colheita para diminuir o risco de deterioração e aumentar o tempo de armazenagem. A modelagem matemática desse processo é importante para a simulação de novas condições operacionais. Com o objetivo de avaliar o perfil da umidade com o tempo de secagem, propõem-se nesse trabalho duas estratégias numéricas para a solução da segunda lei de Fick com coeficiente de difusão variável. Ressalta-se que o coeficiente de difusão é uma função não linear, portanto a equação que descreve o processo de secagem não possui solução analítica. A primeira estratégia consiste em derivar a equação na direção radial empregando a regra do produto e então discretizá-la nesta direção por Diferenças Finitas. Na segunda estratégia, a equação original é discretizada diretamente na direção radial, sendo que para isto são empregadas interpolações lineares para o cômputo das propriedades entre dois pontos da malha. Em ambas as estratégias, após a discretização na direção radial, tem-se um sistema de equações diferencias ordinária (EDOs) que é integrado no tempo utilizando o método de Runge-Kutta de $4^{\circ}$ ordem no software Matlab®. As simulações são realizadas, utilizando-se as condições experimentais reportadas por Barrozo et al. (2006), que resolve o modelo por Colocação Ortogonal. Os resultados das duas estratégias são comparados com dados experimentais. Estas comparações indicam que as duas estratégias são eficientes na simulação da secagem da soja, entretanto a primeira estratégia proporciona uma convergência mais rápida.

Palavras-chave: Soja, secagem, diferenças finitas.

\footnotetext{
${ }^{1}$ UFES - Universidade Federal do Espírito Santo

${ }^{2}$ UFES - Universidade Federal do Espírito Santo

${ }^{3}$ UFES - Universidade Federal do Espírito Santo

${ }^{4}$ UFES - Universidade Federal do Espírito Santo
}

ENGEVISTA, V. 18, n. 1, p. 207-224, Julho 2016. 
ISSN: $1415-7314$

ISSN online: $2317-6717$

\begin{abstract}
The soybean needs to be dried after harvest to decrease the risk of deterioration and to increase the storage time. The mathematical modeling of this process is important for the simulation of new operating conditions. Two numerical strategies for the solution of the second Fick's law with variable diffusion coefficient are proposed in this paper with the objective to assess the moisture profile during drying. It is noteworthy that, the diffusion coefficient is a nonlinear function, therefore the equation describing the drying process does not have analytical solution. The first strategy is to derive the equation in the radial direction using the product rule and then discretize this direction by Finite Difference. In the second strategy, the original equation is directly discretized in the radial direction and the properties between two points of the mesh are calculated through linear interpolation. In both strategies, after discretization in the radial direction, the systems of ordinary differential equations (ODEs) resulting are integrated in time using Matlab®. The simulations are performed using the experimental conditions reported by Barrozo et al. (2006), which solves the model by Orthogonal Collocation. The results of two strategies are compared with experimental data. These comparisons indicate that both strategies were effective in the soybean drying simulation, but the first strategy provides faster convergence.
\end{abstract}

Keywords: Soybean, drying, finite differences. 


\section{Introdução}

A soja é a cultura agrícola brasileira que mais cresceu nas últimas três décadas e corresponde a $49 \%$ da área plantada em grãos do país (MINISTÉRIO DA AGRICULTURA, 2014). Os investimentos nessa cultura agrícola fizeram do Brasil o segundo maior produtor mundial de soja (SEAB, 2013).

No período de 2012/2013 a produção brasileira foi de 82 milhões de toneladas, apenas 0,96\% a menos da produção do maior produtor mundial, EUA (USDA, 2014). O grão de soja é um componente essencial na fabricação de rações animais e utilizado na alimentação humana.

$\mathrm{O}$ processo de produção para a obtenção de grãos com alta qualidade torna-se indispensável, uma vez que rachaduras e quebras ocorrem nos grãos. Dessa forma, algumas características mecânicas têm sido estudadas para predição do comportamento dos grãos. A força de compressão e o módulo proporcional de deformidade diminuem com o aumento do teor de água (RIBEIRO et al., 2007). Segundo Acasio (1997) a soja com umidade superior a 13\% em base úmida necessita ser desidratada para diminuir o risco de autodeterioração, impedir o desenvolvimento de fungos e aumentar o tempo de armazenagem.

A secagem pode ser definida como a aplicação de calor sob condições controladas para remover parte da água presente em um produto por evaporação. $\mathrm{O}$ processo envolve a transferência simultânea de calor e de massa, sendo que o transporte de umidade, do interior para a superfície do material, pode ocorrer na forma de líquido e/ou vapor, dependendo do tipo de produto e do percentual de umidade presente (FIOREZE, 2004).

$\mathrm{Na}$ literatura, encontram-se vários modelos para representar o processo de secagem. $\mathrm{O}$ modelo baseado na segunda lei de Fick é bastante utilizado para representar o processo de secagem da soja em camada delgada. Em coordenadas esféricas considerando o fluxo somente na direção radial o modelo de difusão pode ser representado pela equação abaixo (BIRD et al, 2002):

$$
\frac{\partial \mathrm{M}}{\partial \mathrm{t}}=\frac{1}{\mathrm{r}^{2}} \cdot \frac{\partial}{\partial \mathrm{r}}\left(\mathrm{D}_{\mathrm{ef}} \cdot \mathrm{r}^{2} \cdot \frac{\partial \mathrm{M}}{\partial \mathrm{r}}\right)
$$

Onde,

M - umidade em massa seca (kg de água/ kg de matéria seca);

$\mathrm{r}$ - coordenada radial (m) e

$D_{\text {ef }}$ - coeficiente de difusividade $\left(\mathrm{m}^{2} / \mathrm{s}\right)$.

O coeficiente de difusão engloba os efeitos de todos os fenômenos que podem intervir sobre a migração da água. Pode-se entender a difusividade como a facilidade com que a água é removida do material, que varia conforme mudam as condições de secagem (OLIVEIRA et al., 2006). 
A equação pode apresentar solução analítica dependendo das considerações e aproximações aplicadas na estruturação do problema. Uma simplificação é considerar o coeficiente de difusão constante (eq. 2), metodologia adotada por Golmohammadi, et al. (2014), Menezes et al. (2013), Perussello et al. (2012) e Pfeifer et al. (2010). A eq.2 possui solução analítica, representada na eq.3 (CRANCK, 1975).

$$
\begin{gathered}
\frac{\partial \mathrm{M}}{\partial \mathrm{t}}=\mathrm{D}_{\mathrm{ef}}\left(\frac{2}{\mathrm{r}} \cdot \frac{\partial \mathrm{M}}{\partial \mathrm{r}}+\frac{\partial^{2} \mathrm{M}}{\partial \mathrm{r}^{2}}\right) \\
U R=\frac{M-M_{e q}}{M_{0}-M_{e q}}=\frac{6}{\pi^{2}} \cdot \sum_{n=1}^{\infty} \frac{1}{n^{2}} \cdot \exp \left(\frac{D_{e f} \cdot n^{2} \cdot \pi^{2} \cdot t}{R^{2}}\right)
\end{gathered}
$$

Onde,

UR - umidade relativa;

$\mathrm{R}$ - raio da partícula (m);

$\mathrm{M}_{0}$ - é a umidade inicial ( $\mathrm{kg}$ de água/ kg de matéria seca) e

$\mathrm{M}_{\mathrm{eq}}$ - umidade de equilíbrio.

Se o coeficiente de difusão não for considerado constante a eq.1 não possui solução analítica.

Neste contexto a modelagem da secagem da soja é importante na simulação de novas condições operacionais, objetivando-se a otimização do processo. O objetivo deste trabalho é apresentar e comparar duas estratégias de soluções numéricas para o modelo de difusão pelo método de Diferenças Finitas. O modelo de difusão em coordenadas esféricas e os dados experimentais utilizados para a validação deste artigo foram aplicados por Barrozo et al. (2006).

\section{Material e métodos}

Barrozo et al. (2006) faz um estudo da secagem de soja com coeficiente não constante. Para a resolução desse modelo com as respectivas condições iniciais e de contorno, estes autores aplicam a técnica de Colocação Ortogonal para a discretização na direção radial. As equações diferenciais ordinárias resultantes da discretização foram integradas no tempo.

O modelo de difusão em coordenadas esféricas, eq. (1), foi escolhido para representar a difusão na secagem do grão de soja. A condição inicial da umidade na soja e as condições de contorno proposta por Barrozo et al. (2006) estão representadas nas eq. 4, eq.5 e eq. 6 respectivamente.

$$
\begin{gathered}
M(r, 0)=M_{0} \\
\frac{\partial M}{\partial r}(0, t)=0
\end{gathered}
$$


Para a camada externa da soja a resistência por transferência de massa é insignificante. Portanto é adequado se afirmar que a umidade na superfície da soja atinge instantaneamente a umidade de equilíbrio $\left(\mathrm{M}_{\mathrm{eq}}\right)$ (BARROZO et al., 2006).

$$
\begin{gathered}
\mathrm{M}(\mathrm{R}, \mathrm{t})=\mathrm{M}_{\mathrm{eq}} \\
M_{e q}=\left(\frac{-\exp \left(-0.0067 T_{S}+3.02\right)}{\ln (R H)}\right)^{\frac{1}{1.508}}
\end{gathered}
$$

Onde,

RH - umidade relativa do gás.

Como a difusividade depende da umidade se faz necessário uma equação que represente esse fenômeno. A equação escolhida é proposta por Parti e Dugmanics (1990).

$$
D_{\text {ef }}=\left(\text { a. } \exp \left(\frac{-E}{\text { Rg.T }}\right) \exp (c \mathrm{M})\right) \mathrm{R}^{2}
$$

Onde,

a - parâmetro da eq. $6\left(\mathrm{~s}^{-1}\right)$;

E - energia de ativação $\left(\mathrm{m}^{2} \cdot \mathrm{s}^{-2}\right)$;

$\mathrm{R}_{\mathrm{g}}$ - constante universal dos gases $\left(\mathrm{m}^{2} \cdot \mathrm{s}^{-2} \cdot \mathrm{K}^{-1}\right)$;

$\mathrm{T}$ - temperatura $(\mathrm{K}) \mathrm{e}$

c - parâmetro da eq. 8.

Nesse artigo o modelo foi resolvido por duas estratégias de soluções diferentes. Nas duas estratégias utilizou-se o método das linhas. Esse método procede em dois passos, primeiro a derivada parcial é aproximada usando diferenças finitas e posteriormente o conjunto de equações diferenciais ordinárias (EDOs) resultante é resolvido (WOUWER et al., 2001).

Na primeira estratégia utilizou-se a regra do produto na eq.1, antes da discretização na direção radial por Diferenças Finitas. Na segunda estratégia, a equação original é discretizada diretamente na direção radial, sendo que para isto são empregadas interpolações lineares para o cômputo das propriedades entre dois pontos da malha. O conjunto EDOs foi integrado no tempo utilizando o método de Runge-Kutta de $4^{\circ}$ ordem no software Matlab.

\subsection{Solução numérica: Estratégia 1}

Conforme já discutido, na primeira estratégia, a solução da eq.1 consiste em inicialmente aplicar a regra do produto para derivação. Isto porque na eq. 1 tem-se o produto de 3 termos dependentes da posição radial $\left(\mathrm{D}_{\mathrm{ef}}, \mathrm{r}^{2}\right.$ e M). $\mathrm{O}$ resultado da aplicação da regra do produto está apresentado nas eq. 9, 10 e 11. 


$$
\begin{gathered}
\frac{\partial}{\partial \mathrm{r}}\left[\mathrm{D}_{\mathrm{ef}} \cdot \mathrm{r}^{2} \cdot \frac{\partial \mathrm{M}}{\partial \mathrm{r}}\right]=\mathrm{D}_{\mathrm{ef}} \cdot \mathrm{r}^{2} \cdot \frac{\partial^{2} \mathrm{M}}{\partial \mathrm{r}^{2}}+\frac{\partial \mathrm{M}}{\partial \mathrm{r}} \cdot \frac{\partial}{\partial r}\left(\mathrm{D}_{\mathrm{ef}} \cdot \mathrm{r}^{2}\right) \\
\frac{\partial}{\partial r}\left[\mathrm{D}_{\mathrm{ef}} \cdot \mathrm{r}^{2}\right]=2 \cdot \mathrm{D}_{\mathrm{ef}} \cdot \mathrm{r}+ \\
\mathrm{r}^{2} \cdot \frac{\partial \mathrm{D}_{\mathrm{ef}}}{\partial \mathrm{M}} \cdot \frac{\partial \mathrm{M}}{\partial \mathrm{r}} \\
\frac{\partial \mathrm{D}_{\mathrm{ef}}(\mathrm{M})}{\partial \mathrm{M}}=3,8 \cdot \mathrm{D}_{\mathrm{ef}}
\end{gathered}
$$

Substituindo as eq. 11, 10 e 9 na eq. 1 obtêm-se a eq. 12 .

$$
\frac{\partial \mathrm{M}}{\partial \mathrm{t}}=\frac{1}{\mathrm{r}^{2}}\left[\left(\mathrm{D}_{\mathrm{ef}} \cdot \mathrm{r}^{2} \cdot \frac{\partial^{2} \mathrm{M}}{\partial \mathrm{r}^{2}}\right)+\left(2 \cdot \mathrm{r} \cdot \mathrm{D}_{\mathrm{ef}} \cdot \frac{\partial \mathrm{M}}{\partial \mathrm{r}}\right)+\left(3,8 \cdot \mathrm{D}_{\mathrm{ef}} \cdot \mathrm{r}^{2} \cdot \frac{\partial \mathrm{M}}{\partial \mathrm{r}} \cdot \frac{\partial \mathrm{M}}{\partial \mathrm{r}}\right)\right]
$$

Simplificando obtêm-se a eq. 13.

$$
\frac{\partial \mathrm{M}}{\partial \mathrm{t}}=\mathrm{D}_{\mathrm{ef}} \cdot\left[\frac{\partial^{2} \mathrm{M}}{\partial \mathrm{r}^{2}}+\frac{\partial \mathrm{M}}{\partial \mathrm{r}} \cdot\left(\frac{2}{\mathrm{r}}+\left(3,8 \cdot \frac{\partial \mathrm{M}}{\partial \mathrm{r}}\right)\right)\right]
$$

As derivadas parciais da umidade com o raio serão resolvidas por Diferenças Finitas. Para os pontos internos, as aproximações de segunda ordem por diferenças finitas centrais para as derivadas da umidade são dadas por:

$$
\begin{gathered}
\frac{\partial^{2} \mathrm{M}}{\partial \mathrm{r}^{2}}=\frac{\mathrm{M}_{(\mathrm{i}+1)}+\mathrm{M}_{(\mathrm{i}-1)}-2 \mathrm{M}_{\mathrm{i}}}{\Delta \mathrm{r}^{2}} \\
\frac{\partial \mathrm{M}}{\partial \mathrm{r}}=\frac{\mathrm{M}_{(\mathrm{i}+1)}+\mathrm{M}_{(\mathrm{i}-1)}}{2 . \Delta \mathrm{r}}
\end{gathered}
$$

Onde,

$\Delta r$ - distância entre dois pontos da malha uniforme proposta (m).

Para o último ponto foi utilizado a condição de contorno representada na eq. 7 e desta forma o valor da umidade é especificado (condição de primeiro tipo). Para a discretização no primeiro ponto foi utilizada a condição de contorno (eq.4) e aplicado o método de Diferenças Finitas para frente com três pontos (eq.16). Esta expressão com três pontos foi empregada por também ser de segunda ordem. Siqueira et al. (2012) aplica essa mesma metodologia para o resfriamento de morango e o valor da umidade no centro do grão, $\mathrm{M}_{1}$, pode ser explicitado em função das umidades nos dois pontos internos adjacentes.

$$
\begin{gathered}
\frac{\partial \mathrm{M}}{\partial \mathrm{r}_{1}}=\frac{4 \cdot \mathrm{M}_{2}-3 \cdot \mathrm{M}_{1}-\mathrm{M}_{3}}{2 \cdot \Delta \mathrm{r}} \\
\mathrm{M}_{1}=\frac{4 \cdot \mathrm{M}_{2}-\mathrm{M}_{3}}{3}
\end{gathered}
$$




\subsection{Solução numérica: Estratégia 2}

A discretização constituiu-se em dividir a malha em $\mathrm{n}$ pontos na direção radial, considerando a condição de simetria da soja. Uma opção para a discretização direta da eq.1 seria inicialmente a aplicação da aproximação da derivada primeira por diferenças finitas centrais nos pontos (i-1) e (i+1):

$$
\begin{aligned}
& \frac{\partial \mathrm{M}}{\partial \mathrm{r}}(\mathrm{i}-1)=\frac{\mathrm{M}_{(\mathrm{i})}-\mathrm{M}_{(\mathrm{i}-2)}}{2 \cdot \Delta \mathrm{r}} \\
& \frac{\partial \mathrm{M}}{\partial \mathrm{r}}(\mathrm{i}+1)=\frac{\mathrm{M}_{(\mathrm{i}+2)}-\mathrm{M}_{(\mathrm{i})}}{2 \cdot \Delta \mathrm{r}}
\end{aligned}
$$

Mais além, as diferenças finitas centrais no ponto (i) aplicadas à derivada primeira em relação ao raio fora dos parênteses na eq. 1 , resulta em:

$$
\frac{\partial}{\partial \mathrm{r}}\left[\mathrm{D}_{\mathrm{ef}} \cdot \mathrm{r}^{2} \cdot \frac{\partial \mathrm{M}}{\partial \mathrm{r}}\right]_{\mathrm{i}}=\frac{\left[\mathrm{D}_{\mathrm{ef}} \cdot \mathrm{r}^{2} \cdot \frac{\partial \mathrm{M}}{\partial \mathrm{r}}\right]_{(\mathrm{i}+1)}-\left[\mathrm{D}_{\mathrm{ef}} \cdot \mathrm{r}^{2} \cdot \frac{\partial \mathrm{M}}{\partial \mathrm{r}}\right]_{(\mathrm{i}-1)}}{2 . \Delta \mathrm{r}}
$$

Substituindo-se as expressões dadas pela eq. (18) e eq. (19) na eq. (20) e rearranjando-se a equação e substituindo-se o resultado na eq. (1), têm-se:

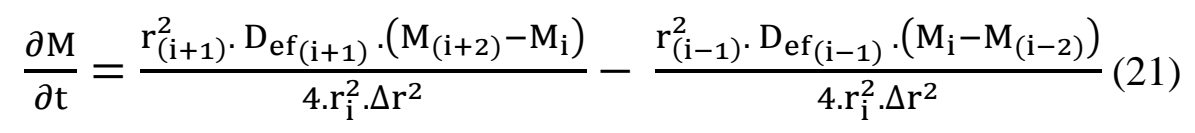

Verifica-se que na eq. 21 para a determinação da derivada temporal no ponto i, são necessários os valores da umidade no ponto e em dois pontos para a direita e dois pontos para a esquerda. Este fato faz com que esta equação não possa ser aplicada nos contornos, como também no segundo e no penúltimo pontos da malha. No segundo ponto, seria necessário o valor da umidade no ponto $(\mathrm{i}=0)$ e no penúltimo ponto seria necessário o valor da umidade no ponto $(\mathrm{i}=\mathrm{n}+1)$. Observa-se que estes pontos não existem e desta forma a eq. 21 não é empregada neste trabalho.

Desta forma, a segunda estratégia de discretização por Diferenças Finitas emprega o cálculo da derivada primeira da umidade em pontos situados entre os pontos da malha. A Figura 1 representa o esquema de discretização adotado nesta segunda estratégia.

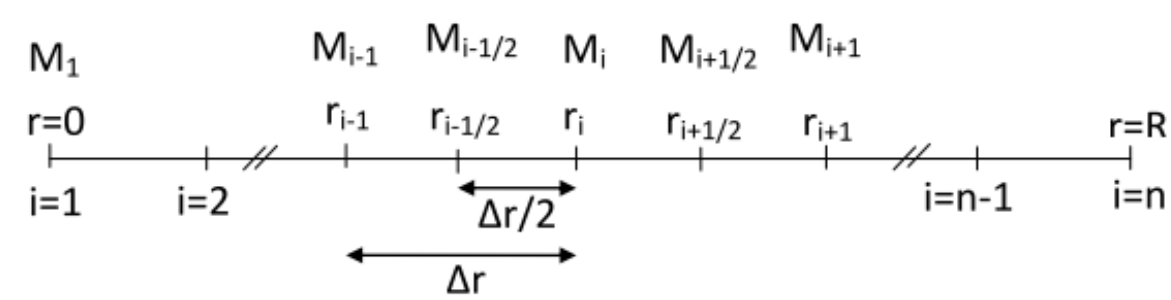

Figura 1- Esquema de discretização para a estratégia 2. 
Para explicar o procedimento adotado, empregamos uma variável auxiliar $\mathrm{U}$, definida como sendo o termo entre parênteses na eq. 1.

$$
\mathrm{U}=\mathrm{D}_{\mathrm{ef}} \cdot \mathrm{r}^{2} \cdot \frac{\partial \mathrm{M}}{\partial \mathrm{r}}
$$

Observa-se que para os pontos internos da malha, a derivada primeira na direção radial da variável U pode ser aproximada por diferenças finitas centrais como:

$$
\frac{\partial U}{\partial r}=\frac{U_{\left(i+\frac{1}{2}\right)}-U\left(i-\frac{1}{2}\right)}{\Delta r}
$$

Substituindo-se esta expressão para a derivada da variável auxiliar U em relação ao raio na eq. 1 , tem-se:

$$
\frac{\partial \mathrm{M}}{\partial \mathrm{t}}=\frac{\left(\mathrm{D}_{\mathrm{ef}} \cdot \mathrm{r}^{2} \cdot \frac{\partial \mathrm{M}}{\partial \mathrm{r}}\right)_{\left(\mathrm{i}+\frac{1}{2}\right)}-\left(\mathrm{D}_{\mathrm{ef}} \cdot \mathrm{r}^{2} \cdot \frac{\partial \mathrm{M}}{\partial \mathrm{r}}\right)_{\left(\mathrm{i}-\frac{1}{2}\right)}}{\mathrm{r}_{\mathrm{i}}^{2} \cdot \Delta \mathrm{r}}(24)
$$

Substituindo-se as eqs. 25 e 26 na eq. 24 obtêm a eq. 27 que representa a equação para a derivada da umidade nos pontos centrais.

$$
\begin{gathered}
\frac{\partial \mathrm{M}}{\partial \mathrm{r}}\left(\mathrm{i}+\frac{1}{2}\right)=\frac{\mathrm{M}_{(\mathrm{i}+1)}-\mathrm{M}_{\mathrm{i}}}{\Delta \mathrm{r}} \\
\frac{\partial \mathrm{M}}{\partial \mathrm{r}}\left(\mathrm{i}-\frac{1}{2}\right) \\
\frac{\partial \mathrm{M}}{\partial \mathrm{t}}=\frac{\mathrm{M}_{\mathrm{i}}-\mathrm{M}_{(\mathrm{i}-1)}}{\Delta \mathrm{r}} \\
\frac{\mathrm{r}_{\left(\mathrm{i}+\frac{1}{2}\right)}^{2} \cdot \mathrm{D}_{\mathrm{ef}}\left(\mathrm{i}+\frac{1}{2}\right)}{\mathrm{r}_{\mathrm{i}}^{2} \cdot \Delta \mathrm{r}^{2}} \cdot\left(\mathrm{M}_{(\mathrm{i}+1)}-\mathrm{M}_{\mathrm{i}}\right) \\
\mathrm{r}
\end{gathered}
$$

Para determinar o raio (eq. 28 e 29) e a umidade (eq. 30 e 31 ) no ponto central, entre dois pontos da malha, foi realizada uma interpolação linear, o que equivale ao cômputo da média entre os pontos.

$$
\begin{gathered}
r_{\left(i+\frac{1}{2}\right)}=\frac{r_{(i+1)}+r_{(i)}}{2} \\
r_{\left(i-\frac{1}{2}\right)}=\frac{r_{(i-1)}+r_{(i)}}{2} \\
M_{\left(i+\frac{1}{2}\right)}=\frac{M_{(i+1)}+M_{(i)}}{2}
\end{gathered}
$$




$$
\mathrm{M}_{\left(\mathrm{i}-\frac{1}{2}\right)}=\frac{\mathrm{M}_{(\mathrm{i}-1)}+\mathrm{M}_{(\mathrm{i})}}{2}
$$

Como a difusividade é uma função da umidade, o valor da mesma é calculado pela eq. 32 e pela eq. 33 .

$$
\begin{aligned}
& D_{e f\left(i+\frac{1}{2}\right)}=8,64 \cdot R^{2} \cdot \exp \left(\frac{-4848 \cdot 5}{T}\right) \cdot \exp \left[3,8 \cdot\left(\frac{M_{(i+1)}+M_{(i)}}{2}\right)\right] \\
& D_{e f\left(i-\frac{1}{2}\right)}=8,64 \cdot R^{2} \cdot \exp \left(\frac{-4848 \cdot 5}{T}\right) \cdot \exp \left[3,8 \cdot\left(\frac{M_{(i-1)}+M_{(i)}}{2}\right)\right]
\end{aligned}
$$

As equações discretizadas para o primeiro e o último ponto se equivalem àquelas adotadas na primeira estratégia e estão representadas nas eqs. 6 e 17, respectivamente.

Os valores das condições operacionais empregadas nas simulações foram os mesmos adotados em Barrozo et al. (2006). Desta forma, foi empregado o raio da soja de $3 \times 10^{-3} \mathrm{~m} \mathrm{e}$ temperaturas de $31,5^{\circ} \mathrm{C}, 35^{\circ} \mathrm{C}$ e $58,5^{\circ} \mathrm{C}$ com suas respectivas umidades do gás, $51 \%, 40 \%$ e $12 \%$. A umidade inicial foi de 0,236, sendo reportada em Costa e Passos (2009).

Após todas as discretizações por Diferenças Finitas a equação diferencial parcial se torna um conjunto de EDOs de primeira ordem, que foi integrado no sofware Matlab utilizando-se a rotina de integração do método de Runge-Kutta. Em todas as simulações realizadas, os valores definidos para as tolerâncias relativa e absoluta de integração foram de $10^{-6}$. Desta forma, na análise dos erros numéricos considera-se que os desvios apresentados são provenientes das aproximações realizadas nas discretizações. Esta suposição é razoável já que as ordens de grandeza dos erros numéricos são bastante superiores aos valores estipulados para as tolerâncias de integração no tempo.

\section{Resultados e discussão}

Para demonstrar a aplicabilidade do modelo proposto à secagem da soja, inicialmente são comparados os valores simulados, dos perfis temporais da razão de umidade média no grão, com os valores experimentais apresentados em Barrozo et al. (2006). Esta razão de umidade, UR, é definida na eq.34.

Onde,

$$
U R=\frac{\bar{M}-M_{e q}}{M_{0}-M_{e q}}
$$

Mo - umidade inicial do grão, antes do início do processe de secagem (kg de água/ kg de matéria seca);

Meq - umidade de equilíbrio do grão com o ar na temperatura considerada (kg de água/ kg de matéria seca) e

$\bar{M}$ - umidade média no grão (kg de água/ kg de matéria seca). 
O emprego da razão de umidade é interessante porque a mesma é uma variável adimensional, sendo que seu valor é unitário no início do processo de secagem e tenderia a zero se o tempo de secagem fosse muito grande (na prática, por razões econômicas não se seca até a umidade de equilíbrio).

Para calcular a umidade média no grão em cada tempo, a partir dos dados de umidade em cada ponto da malha, foi empregada a eq.35.

$$
\bar{M}=\frac{\sum_{\mathrm{i}=1}^{\mathrm{n}-1}\left[\mathrm{r}(\mathrm{i})^{2}+\mathrm{r}(\mathrm{i}+1)^{2}\right] \cdot[\mathrm{M}(\mathrm{t}, \mathrm{i})+\mathrm{M}(\mathrm{t},(\mathrm{i}+1)]}{2 \cdot \sum_{\mathrm{i}=1}^{\mathrm{n}-1}\left[\mathrm{r}(\mathrm{i})^{2}+\mathrm{r}(\mathrm{i}+1)^{2}\right]}
$$

A proposta desta eq. 35 é calcular, no numerador, a umidade em todo o grão somando-se as umidades contidas em cada casca de soja correspondente ao intervalo entre dois pontos da malha. Dentro destas cascas, o valor da umidade foi assumido como sendo a média aritmética das umidades nos extremos. Embora a espessura dos intervalos seja constante, o raio não é e por isto também foi computado um valor médio das áreas superficiais das partes interior e exterior de cada casca. Finalmente os valores das umidades de cada casca são ponderados pelos volumes das mesmas e divididos pelo volume total do grão, calculado no denominador. Ressalta-se que na eq. 35 foram simplificadas as constantes que surgem no numerador e denominador, a saber, $\pi, \Delta \mathrm{r}$ (espessura utilizada no cálculo dos volumes das cascas) e a divisão por 2 no cômputo do raio ao quadrado médio.

As Figuras 2, 3 e 4 apresentam as comparações entre os perfis temporais de UR, experimentais e simulados, empregando-se a estratégia 1 e a estratégia 2. Para que nessa análise os erros numéricos associados às aproximações por diferenças finitas fossem desprezíveis, foram empregadas malhas com 1000 pontos (a partir deste valor, um acréscimo no número de pontos de discretização as modificações na solução numérica são desprezíveis para ambas as estratégias e a solução foi considerada convergida).

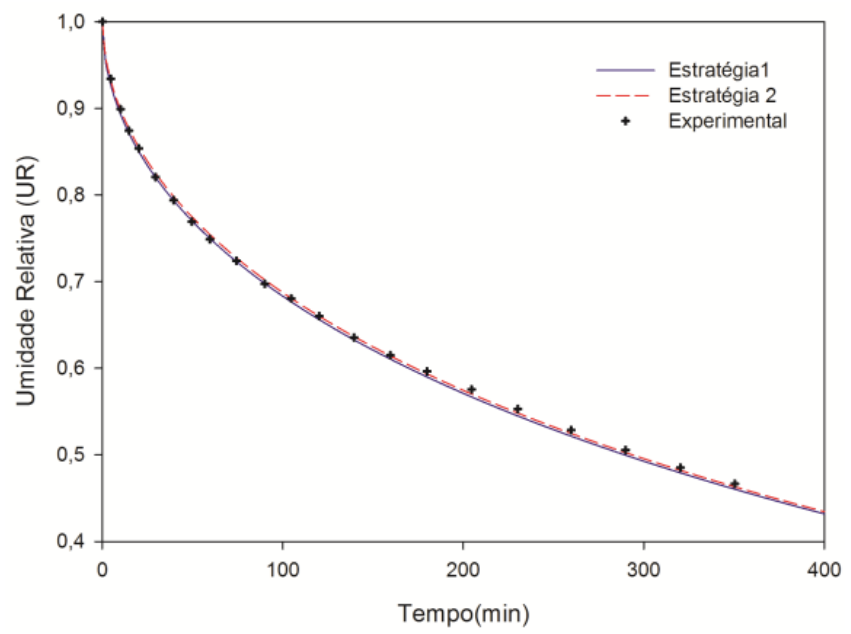

Figura 2 - Umidade relativa considerando os dados da estratégia 1, estratégia 2 e os dados experimentais (temperatura $31,5^{\circ} \mathrm{C}$ ). 


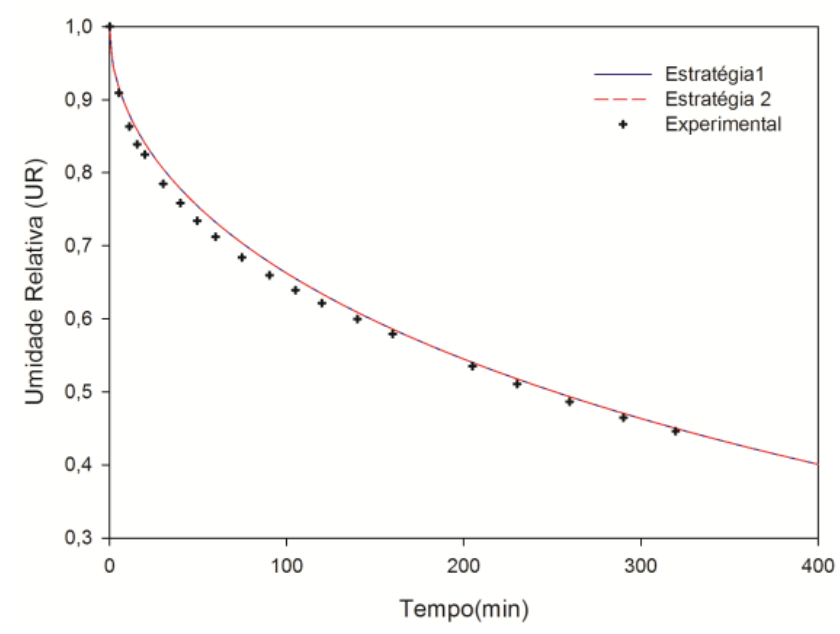

Figura 3 - Umidade relativa considerando os dados da estratégia 1, estratégia 2 e os dados experimentais (temperatura $35^{\circ} \mathrm{C}$ ).

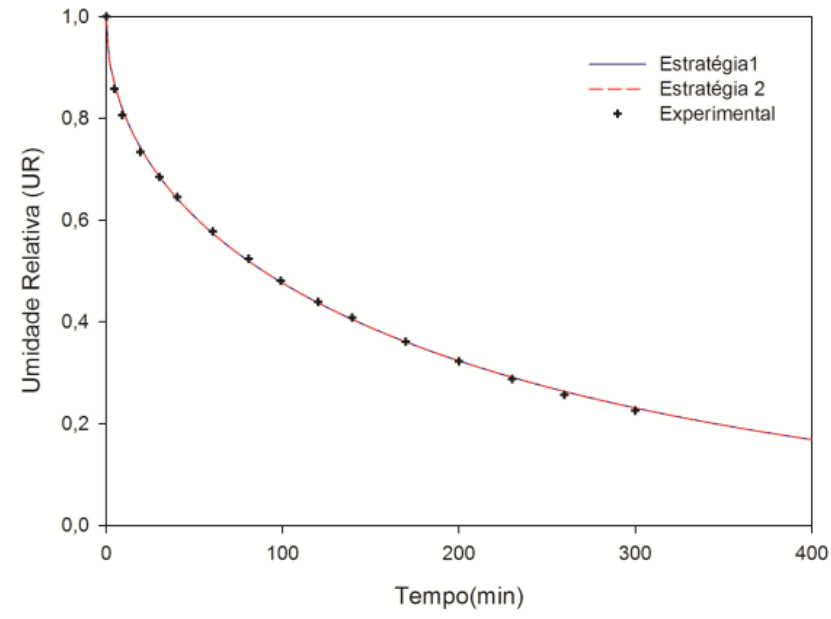

Figura 4 - Umidade relativa considerando os dados da estratégia 1, estratégia 2 e os dados experimentais (temperatura $58,5^{\circ} \mathrm{C}$ ).

Verifica-se nas Figuras 2 a 4 que ambas as estratégias de discretização fornecem a mesma solução convergida. Mais além, a concordância entre os dados simulados e experimentais demonstra a adequação do modelo proposto e confirma as conclusões de Barrozo et al. (2006).

As análises gráficas de convergência da malha para diferentes temperaturas são apresentadas na Figura 5 (estratégia 1) e na Figura 6 (estratégia 2). 
ISSN: $1415-7314$

ISSN online: 2317-6717

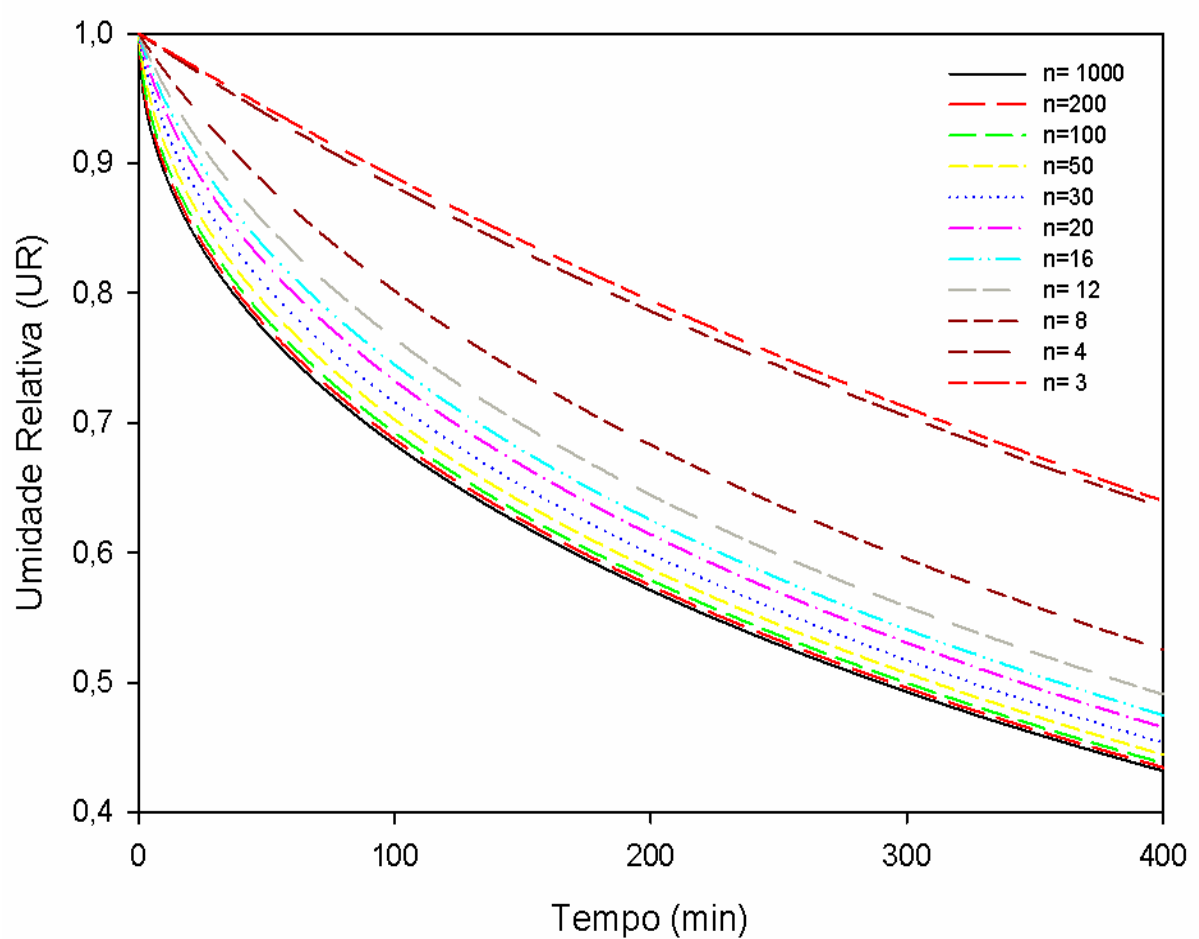

(a)

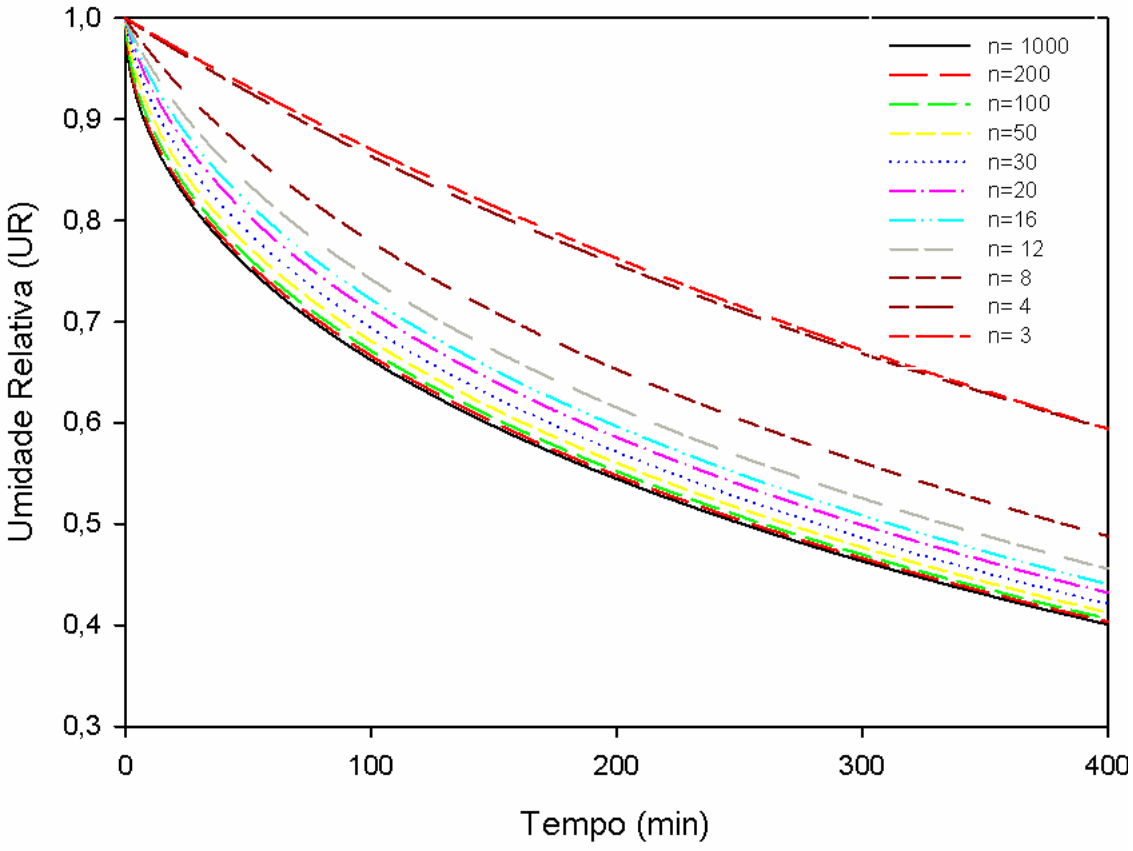

(b) 
ISSN: $1415-7314$

ISSN online: 2317-6717

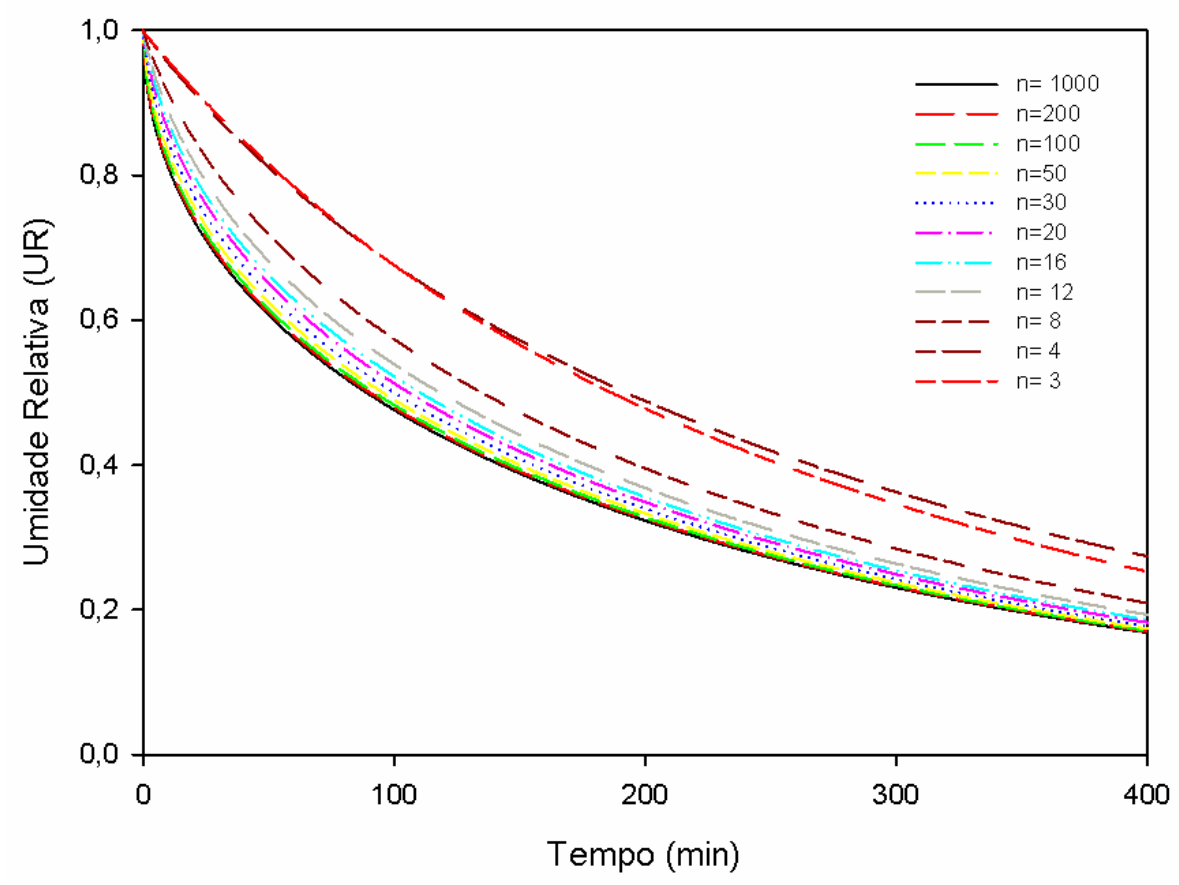

(c)

Figura 5 - Comparação dos perfis temporais da umidade na soja com a solução convergida para estratégia 1, nas temperaturas: (a) 31,5 , (b) 35 e (c) $58,5^{\circ} \mathrm{C}$.

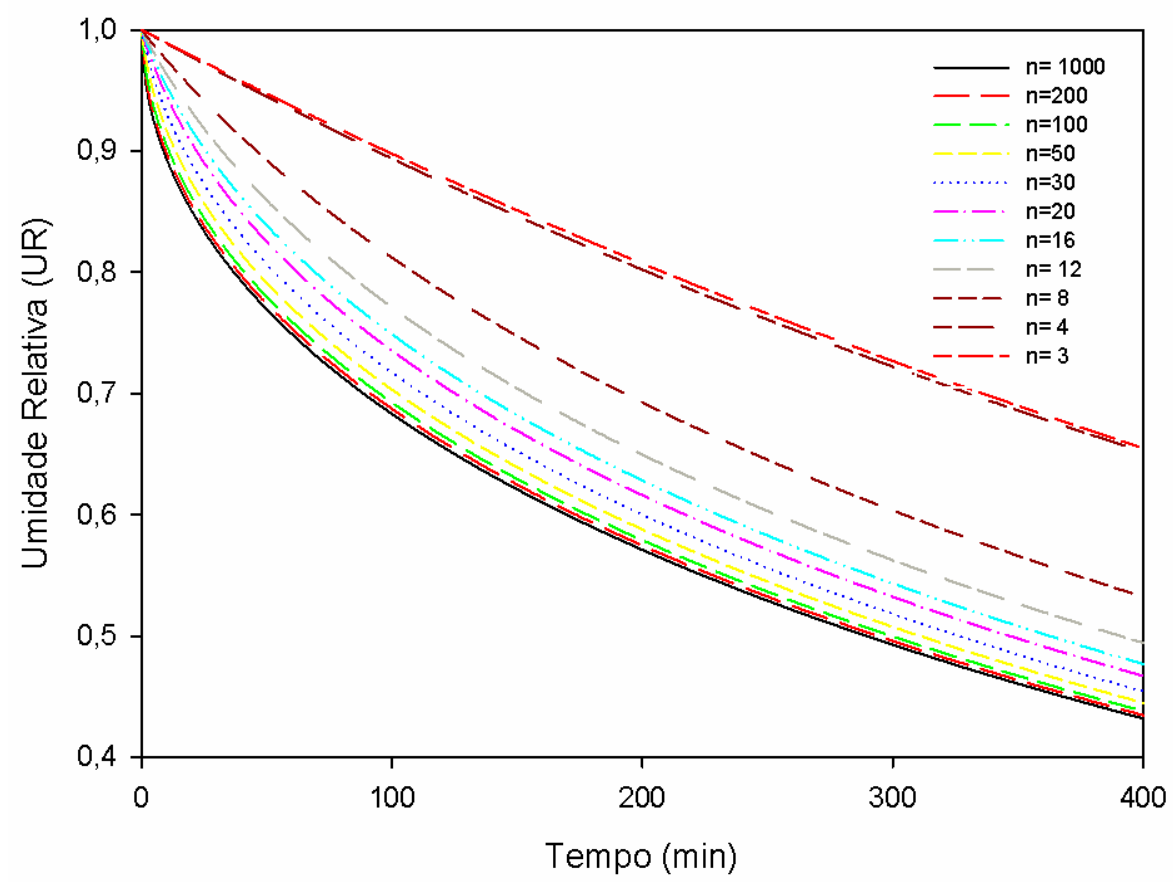

(a) 


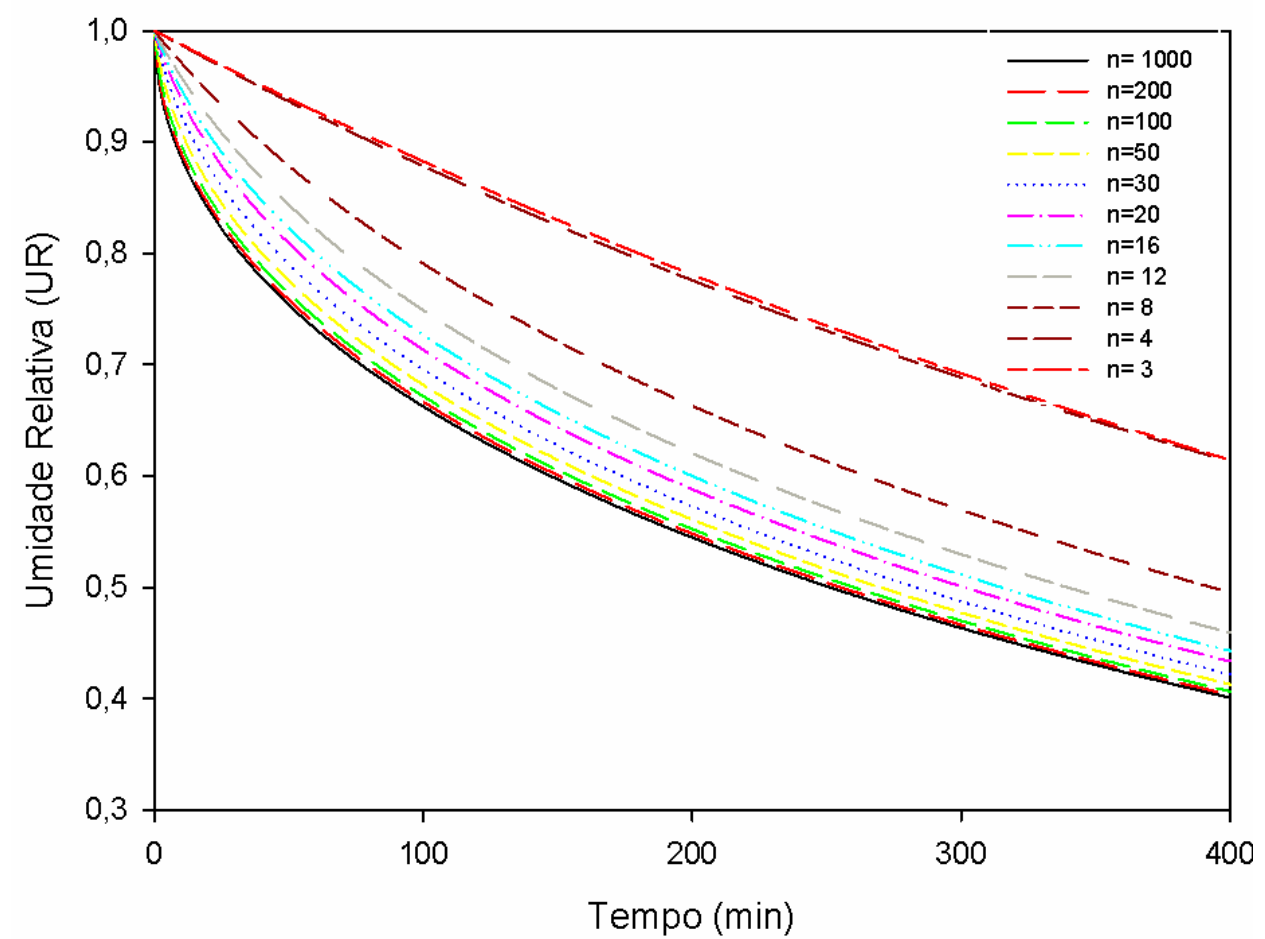

(b)

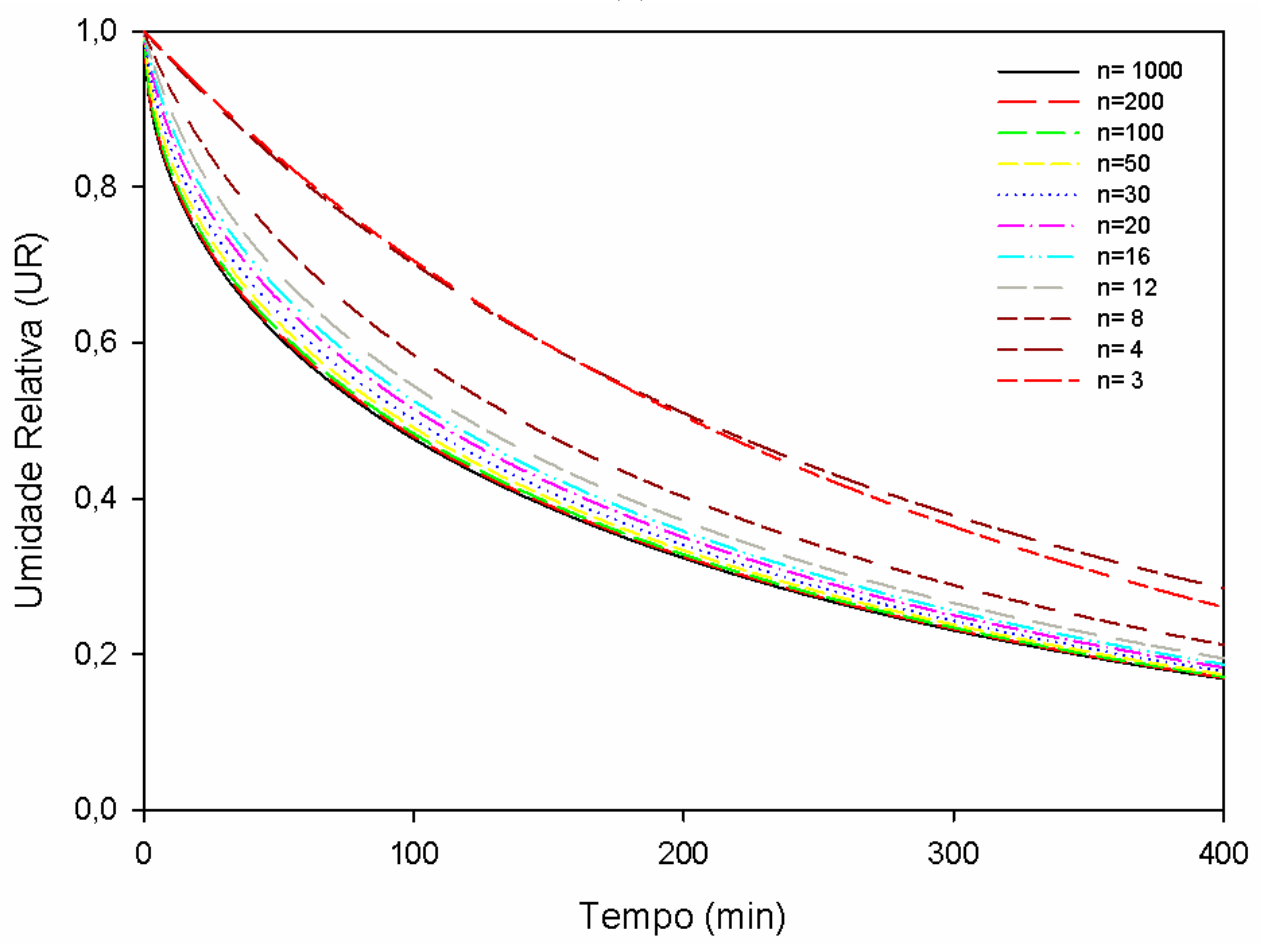

(c)

Figura 6 - Comparação dos perfis temporais da umidade na soja com a solução convergida para estratégia 2 , nas temperaturas: (a) 31,5 , (b) 35 e (c) $58,5^{\circ} \mathrm{C}$.

Pode-se verificar nas Figuras 5 e 6 que, para ambas as estratégias, a solução obtida converge com o refinamento malha. Com a proposta de avaliar a taxa de convergência das duas estratégias de solução numéricas, utilizou-se somatório dos erros ao quadrado (eq. 36), considerando-se os valores de razão de umidade calculados até os 400 minutos de operação. 
Onde,

$$
E R R O=\sum_{i=1}^{400}\left(M R_{n}(i)-M R_{c}(i)\right)^{2}
$$

$\mathrm{MR}_{\mathrm{n}}(\mathrm{i})$ - razão de umidade calculada numericamente;

$\mathrm{MR}_{\mathrm{c}}(\mathrm{i})$ - razão de umidade da solução convergida e $\mathrm{i}$ - tempo de simulação (min).

As Tabelas 1, 2 e 3 apresentam os valores calculados dos erros para diferentes números de pontos na malha.

Tabela 1 - Erro para a estratégia 1 e 2 (temperatura de $31,5^{\circ} \mathrm{C}$ ).

\begin{tabular}{ccc}
\hline $\begin{array}{c}\text { Número de } \\
\text { pontos }\end{array}$ & Estratégia 1 & Estratégia 2 \\
\hline 200 & $3,17 \times 10^{-3}$ & $3,25 \times 10^{-3}$ \\
150 & $6,34 \times 10^{-3}$ & $6,53 \times 10^{-3}$ \\
100 & $1,59 \times 10^{-2}$ & $1,65 \times 10^{-2}$ \\
50 & $7,00 \times 10^{-2}$ & $7,44 \times 10^{-2}$ \\
30 & $2,00 \times 10^{-1}$ & $2,17 \times 10^{-1}$ \\
20 & $4,53 \times 10^{-1}$ & $5,04 \times 10^{-1}$ \\
18 & $5,60 \times 10^{-1}$ & $6,26 \times 10^{-1}$ \\
16 & $7,08 \times 10^{-1}$ & $7,97 \times 10^{-1}$ \\
14 & $9,23 \times 10^{-1}$ & 1,05 \\
12 & 1,25 & 1,43 \\
10 & 1,79 & 2,07 \\
8 & 2,76 & 3,23 \\
6 & 4,74 & 5,58 \\
4 & 9,54 & 1,10 \\
3 & $1,02 \times 10^{1}$ & $1,14 \times 10^{1}$ \\
\hline
\end{tabular}

Tabela 2 - Erro para a estratégia 1 e 2 (temperatura de $35^{\circ} \mathrm{C}$ )

\begin{tabular}{ccc}
\hline $\begin{array}{c}\text { Número de } \\
\text { pontos }\end{array}$ & Estratégia 1 & Estratégia 2 \\
\hline 200 & $2,94 \times 10^{-3}$ & $3,02 \times 10^{-3}$ \\
150 & $5,89 \times 10^{-3}$ & $6,08 \times 10^{-3}$ \\
100 & $1,48 \times 10^{-2}$ & $1,54 \times 10^{-2}$ \\
50 & $6,51 \times 10^{-2}$ & $6,94 \times 10^{-2}$ \\
30 & $1,86 \times 10^{-1}$ & $2,03 \times 10^{-1}$ \\
20 & $4,23 \times 10^{-1}$ & $4,73 \times 10^{-1}$ \\
18 & $5,22 \times 10^{-1}$ & $5,89 \times 10^{-1}$ \\
16 & $6,61 \times 10^{-1}$ & $7,51 \times 10^{-1}$ \\
14 & $8,63 \mathrm{E}^{-1}$ & $9,89 \times 10^{-1}$ \\
12 & 1,17 & 1,36 \\
10 & 1,68 & 1,97 \\
8 & 2,60 & 3,09 \\
6 & 4,51 & 5,42 \\
4 & 9,26 & $1,09 \times 10^{1}$ \\
3 & 9,71 & $1,13 \times 10^{1}$ \\
\hline
\end{tabular}


Tabela 3 - Erro para a estratégia 1 e 2 (temperatura de $58,5^{\circ} \mathrm{C}$ )

\begin{tabular}{ccc}
\hline $\begin{array}{c}\text { Número de } \\
\text { pontos }\end{array}$ & Estratégia 1 & Estratégia 2 \\
\hline 200 & $1,45 \times 10^{-3}$ & $1,48 \times 10^{-3}$ \\
150 & $2,90 \times 10^{-3}$ & $2,99 \times 10^{-3}$ \\
100 & $7,31 \times 10^{-3}$ & $7,61 \times 10^{-3}$ \\
50 & $3,25 \times 10^{-2}$ & $3,47 \times 10^{-2}$ \\
30 & $9,43 \times 10^{-2}$ & $1,03 \times 10^{-1}$ \\
20 & $2,18 \times 10^{1}$ & $2,45 \times 10^{-1}$ \\
18 & $2,71 \times 10^{-1}$ & $3,07 \times 10^{-1}$ \\
16 & $3,45 \times 10^{-1}$ & $3,95 \times 10^{-1}$ \\
14 & $4,55 \times 10^{-1}$ & $5,26 \times 10^{-1}$ \\
12 & $6,27 \times 10^{-1}$ & $7,33 \times 10^{-1}$ \\
10 & $9,17 \times 10^{-1}$ & 1,09 \\
8 & 1,47 & 1,78 \\
6 & 2,70 & 3,34 \\
4 & 6,33 & 7,88 \\
3 & 5,81 & 7,55 \\
\hline
\end{tabular}

Observa-se nas Tabelas de 1 a 3 que, para malhas com mais que 100 pontos, os erros são pequenos em todos os casos e ambas as estratégias possibilitam a convergência da solução com o refinamento da malha. Comparando-se os erros obtidos para malhas com mesmo número de pontos, em diferentes temperaturas, o erro é menor para valores de temperaturas mais elevados. Este fato acontece porque a secagem é mais rápida para temperaturas maiores e desta forma os valores de razão de umidade são menores.

Comparando-se as duas estratégias apresentadas, a estratégia 1 apresentou menor erro quando comparada com a estratégia 2 para todos os testes feitos. Pode-se concluir que a metodologia 1 fornece uma convergência mais rápida do que a metodologia 2. Entretanto, nesta primeira estratégia, todo o procedimento de determinação das equações discretizadas deve ser refeito caso se deseje empregar uma nova correlação para o coeficiente de difusão, o que não acontece na segunda estratégia.

\section{Conclusões}

A secagem é um processo muito importante no armazenamento e processamento dos grãos de soja. O conhecimento da cinética de secagem da soja é essencial para o controle de qualidade dos grãos durante o processo de secagem. O desenvolvimento de um modelo matemático que descreva a cinética do mecanismo de fluxo interno por difusão é importante, pois determinações experimentais podem ser custosas e complexas. Entretanto somente a 
determinação do modelo em muitos casos não é o suficiente quando o mesmo não possui solução analítica. Portanto encontrar um método numérico adequado de solução se faz necessário.

As duas metodologias de solução do modelo aplicadas nesse trabalho, tiveram uma boa convergência e são adequadas para representar o processo de transferência de massa na secagem da soja. Entretanto a primeira estratégia apresenta uma convergência mais rápida, contudo esta metodologia é custosa, pois caso se deseje empregar uma nova correlação para o coeficiente de difusão o procedimento de determinação das equações discretizadas deve ser refeito.

Essa mesma metodologia pode ser aplicada não somente em um problema de transferência de massa, mas também para um problema de transferência de calor com coeficiente variável.

\section{Agradecimento}

Os autores agradecem à CAPES pelas bolsas de mestrado, ao CNPq pela bolsa de produtividade DTI e à FAPES pela bolsa pesquisador capixaba. 


\section{REFERÊNCIAS}

ACASIO, U. A. 1997. Handling and Storage Of Soybeans and Soybean Meal. Feedmachinery [Online]. Available:http://www.feedmachinery.com/articles/feed technology/soybean storage1/ [Accessed 16 Dezembro 2014].

BIRD, R. B.; STEWART, W. E. \& Lightfoot, E. N. 2002. Transport Phenomena. New York, John Wiley.

BARROZO, M.A.S., HENRIQUE H.M., SARTORI, D.J.M. \& FREIRE, J.T. 2006. The use of the orthogonal collocation method on the study of the drying kinetics of soybean seeds. Stored Products Research, 42, 348-356.

COSTA, E. F. Jr \& PASSOS, M. L. 2009. Simulação da secagem transiente de sólidos particulados em leitos de jorro. XXXIV Congresso Brasileiro de Sistemas Particulados. Campinas-SP, Brasil.

CRANK, J. 1975. The mathematics of diffusion, Oxford, Oxford University Press.

FIOREZE, R. 2004. Princípios de secagem de produtos biológicos. João Pessoa, PB. Editora Universitária da UFPB.

GOLMOHAMMADI, M., ASSAR, M., HAMANEH, M. R. \& HASHEMI, S.J. 2014. Energy efficiency investigation of intermittentpaddy rice dryer: Modeling and experimental study. Food and Bioproducts Processing, xxx, 1-9.

MENEZES, M. L. D., STRÖHER, A. P., PEREIRA, N. C. \& BARROS, S. T. D. 2013. Análise da cinética e ajustes de modelos matemáticos aos dados de secagem do bagaço do maracujáamarelo. Engevista,15, 176-186.

MINISTÉRIO DA AGRICULTURA $2014 . \quad$ Soja. [Online]. Available:http://www.agricultura.gov.br/vegetal/culturas/soja/saiba-mais. [Accessed 30 novembro 2014].

OLIVEIRA, R. A. D., OLIVEIRA, W. P. D. \& PARK, K. J. 2006. Determinação da difusividade efetiva de raiz de chicória. Engenharia Agricola, 26, 181-189.

PERUSSEllO, C. A., MARIANI, V. C. \& AMARANTE, Á. C. C. D. 2012. Numerical and experimental analysis of the heat and mass transfer during. Applied Thermal Engineering, 48, 325-331.

PFEIFER, A. A., MURATA, V. V. \& BARROZO, M. A. S. 2010. Modelling of soybean seed drying in concurrent sliding bed dryers: Effect of the number of stages on the seed quality and drying performance. Biosystems engineering, 107, 341-348.

RIBEIRO, D. M. , CORREA, P. C., FURTADO, B. F., GONELI, A. D. \& RESENDE, O. 2007. Propriedades mecânicas dos grãos de soja em função do teor de água. Engenharia Agrícola, 27, 493-500.

SEAB -SECRETARIA DE ESTADO DA AGRICULTURA E DO ABASTECIMENTO. 2013. Soja - Análise da Conjuntura Agropecuária, DERAL - Departamento de Economia Rural, 1-17. SIQUEIRA, A. J. H., COSTA, A. O. \& COSTA Jr. E. F. 2012. Modeling and simulation of forcedair cooling of strawberries using variable convective coefficient. Engenharia Agrícola, 32, 164173.

USDA-UNITED STATES DEPARTAMENT OF AGRICULTURE. 2014. Foreign Agricultural Service [Online], Available: http://apps.fas.usda.gov/psdonline/psdQuery.aspx..[Accessed 15 dezembro 2014].

WOUWER, A. V., SAUCEZ, Ph. \& SCHIESSER, W.E. 2001. Adaptive Method of Lines. Florida, Chapman \& Hall. 